\title{
A School-Based Cluster Randomized Controlled Trial to Evaluate the Effectiveness of Nutrition Behavior Change Communication Interventions in Improving Nutritional Status and Academic Performance of Schoolchildren in South Ethiopia: Study Protocol
}

\section{Tsedeke Wolde* and Tefera Belachew}

Nutrition Unit, Department of Population and Family Health, College of Health Sciences, Jimma University, Jimma, Ethiopia

*Corresponding author: Tsedeke Wolde, Nutrition Unit, Department of Population and Family Health, College of Health Sciences, Jimma University, Jimma, Ethiopia, Tel: +25910943068; E-mail: tswmh2006@gmail.com

Received date: April 26, 2018; Accepted date: December 17, 2018; Published date: December 24, 2018

Copyright: (c) 2018 Wolde T, et al. This is an open-access article distributed under the terms of the Creative Commons Attribution License, which permits unrestricted use, distribution, and reproduction in any medium, provided the original author and source are credited.

\begin{abstract}
Background: School aged children in developing countries are vulnerable to malnutrition, which leads to stunting and can have adverse effects on mental development and academic performance at school. Despite several decades of work in improving childhood nutrition in Ethiopia, stunting remains a major public health concern with lack of evidence on its effect on school performance. The primary aim of this study is to addresses this gap by evaluating the effect of behavior change communication intervention on nutritional status and academic performance among school aged children in Meskan District of South Ethiopia.
\end{abstract}

Methods: This school-based cluster randomized trial (c-RCT) compares the intervention arm with control arm. The intervention arm receives behavior change communication (BCC) on essential nutrition actions. The control arm receives only routine health and nutrition services. From a Meskan District, 10 clusters were selected and randomly assigned to the two study arms by randomization. The study participants will be children 10-15 years of age. Total sample size was 408 study participants. Anthropometric measurements, academic performance, nutritional knowledge, and dietary diversity will be measured at both baseline and end line. A behavior change and key poster message was providing based on essential nutrition actions for science teachers and students using class sessions and school visits. A binary logistic regression analysis will be used to identify independent predictors of nutritional status and academic performance of schoolchildren after controlling for confounding variables. The effect of BCC on micronutrient will be determined using generalized estimating equations (GEE) and or linear mixed effects models.

Results: A total of 378 primary schoolchildren (10-15 years of age) were involved in the study giving a response rate of $93 \%$. The mean age of the students was 12.8 years (SD \pm 1.3 years). The prevalence of stunting and underweight was $16.9 \%$ and $37.3 \%$ respectively. The mean score of overall grades was $64.52 \pm 8.45$.

Discussion: The findings of this study will provide sufficient evidence to develop policies and programs aimed to improve dietary diversity in children as well as nutritional knowledge (10-15 years) and to prevent micronutrient deficiencies and poor academic performance in school settings. It will also provide recommendations for strengthening the school nutrition component of behavior change communication in child health and nutrition programs.

Trial registration: The study was retrospectively registered on October 28, 2016 and is available online at ClinicalTrials.gov (ID: NCT02956941).

Keywords: Randomized controlled trial; Nutrition BCC interventions; Academic performance; Nutritional status; School aged children; South Ethiopia

Abbreviations: BCC: Behavior Change Communication; BMI: Body Mass Index; CSA: Central Statics Agency; CONSORT: Consolidated Standards of Reporting Trials; CC: Control Cluster; EDHS: Ethiopian Demographic and Health Survey; FAO: Food and Agriculture Organization; GEE: Generalized Estimating Equation; HFA: Height for Age; ICC: Intra-Cluster Correlation Coefficient; IRB: Institutional Review Board; IC: Intervention Cluster; Kg: Kilogram; MOH: Ministry of Health; NNP: National Nutrition Program; SD: Standard Deviation;
SPSS: Statistical Package for Social Science; WHO: World Health Organization.

\section{Background}

The estimated prevalence of chronically malnourished (stunted) children around the world was 178 million. Of the estimated 178 million, $90 \%$ live in 36 countries, one of which is Ethiopia [1]. Chronic malnutrition (stunting) continues to be a major public health problem in developing countries including Ethiopia. Over the past decade, Ethiopia has shown encouraging progress in reducing malnutrition. The baseline levels of chronic malnutrition in this country still remain 
Citation: Wolde T, Belachew T (2018) A School-Based Cluster Randomized Controlled Trial to Evaluate the Effectiveness of Nutrition Behavior Change Communication Interventions in Improving Nutritional Status and Academic Performance of Schoolchildren in South Ethiopia: Study Protocol. J Nutr Disorders Ther 8: 240. doi:10.4172/2161-0509.1000240

Page 2 of 9

so high and much effort is expected to be applied. Ethiopia must continue to make significant investments in nutrition [2]. Thus, if intervention is not taken, the problem goes through adolescence and adulthood [2].

Protein energy is essential for brain development, so protein energy malnutrition in early childhood negatively affects intellectual development and results low academic performance in late adolescence. Chronic malnutrition in all forms causes problems like illness, brain damage, delayed physical growth, delayed development of motor skills and delayed intellectual development that have consequence directly or indirectly on school performance $[3,4]$.

A study led by the African Union, the Cost of Hunger in Africa (COHA), is a multi-country study aimed at estimating the economic and social impacts of under nutrition in Africa states $16 \%$ of al repetitions in primary school are associated with stunting, stunted children achieve 1.1 years less in school education. Ethiopia's workforce by $8 \%$ and the total cost of malnutrition on education, productivity and health is estimated to be Ethiopian Birr (ETB) 55.5 billion, which is equivalent to $16.5 \%$ of GDP $[1,5]$.

Poor academic achievement has been a major area of concern for educators, parents, and school children for more than three decades [6].

There are several reasons for children to underperform at school. Including; medical problems, below average intelligence, specific learning disabilities, attention deficit hyperactivity disorder, emotional problems, a poor sociocultural home environment, psychiatric disorders, or environmental factors [7]. Nutritional problems are among major factors that affect learning and school performance. Several studies have explored the relationship between nutritional anthropometric indicators and school performance indicators, such grade level, age at enrollment, absenteeism, achievement test scores, intelligence quotient (IQ), and performance on selected cognitive tasks, including concentration in the classroom. Many studies revealed significant findings between the nutritional status indicators and cognitive test scores or school performance indicators [8].

According to Ethiopia National Nutrition Program (NNP) baseline survey conducted in 2010,23\% of adolescent girls were stunted and $9 \%$ were severely stunted. Girls 13-14 were more likely to be stunted than older girls likewise, girls living in rural areas $(28 \%)$ were more likely to be stunted than girls living in urban areas (12\%) [9].

Study conducted in West Ethiopia indicates that prevalence of stunting was $17 \%$. Severely stunted adolescents were $1.7 \%$ [10]. Furthermore, cross sectional study in Eastern Ethiopia shows that the prevalence of stunting was $25.5 \%$. Stunting was more prevalent among males and early adolescents [11]. Another study was conducted among 790 primary school children from rural Ethiopia that children from a family size of 6-8 were more likely to be stunted than those from family size of 2-5 members [12].

In Northeast Ethiopia, the overall prevalence of stunting among the adolescents (10-19 years) was $15.5 \%$. After possible potential confounders were controlled; being male, being in the age group 13-16 using unsafe drinking water supply and having no latrine facility were found significantly associated with stunting [13]. A school based crosssectional study in Northwest Ethiopia had revealed the overall prevalence of stunting among adolescent girls was $33.1 \%$. Middle age of adolescence, unsatisfactory media exposure and poor mother's education were significantly associated with stunting $[14,15]$.
The 5-year longitudinal study in Jimma zone, southwest Ethiopia shows that highest grade completed, household income and job were positively associated with height for age z-score. However, male gender, last attended in community school, abdominal pain and household size were negatively associated with Height for age z-score [16]. In Ethiopia, a study in Addis Ababa among high school adolescents showed that the prevalence of stunting (low height-for-age) in all school adolescents was 7.2\%. Adolescents in government schools were significantly more likely to be undernourished [stunting; $51(10.0 \%)$ versus $23(4.5 \%)$ compared to their counterparts in private schools [17].

Although chronic malnutrition is a public health problem in Ethiopia, there is little documented information on the nutritional status of schoolchildren and adolescents and the impact on their academic performance in Southern Ethiopia. Recently, the Ethiopian Ministry of Education recognized the impact of poor health and nutrition on children's ability to learn, their school attendance, and concentration. In response, Ministry of Education developed the National School Health and Nutrition Strategy (2012) emphasizing health and nutrition to improve student scholastic performance $[18,19]$.

The future of a nation entirely depends on the welfare of its younger generation. Ethiopia is currently transitioning from the end of its first national growth and transformation plan (GTP I) to the beginning of its second growth and transformation plan (GTP II). Its success will largely depend on the academic performance of its schoolchildren and their future contributions [19]. GTP II and the Fifth Education Sector Development Programme (ESDP V) began in 2015, when national gross enrolment rate (GER) in primary school (grade $5-8$ ) was $71.1 \%$ and $74.2 \%$ in Southern Nations, Nationalities and Peoples Region (SNNPR). The national repetition rate among primary schoolchildren was $6.7 \%$, slightly higher in males $(7.1 \%)$ than females $(6.2 \%)$. The national dropout rate was $10.1 \%$ [20].

Behavioral change communication (BCC) plays a vital role in providing knowledge, changing people's attitudes and norms [21]. BCC is the strategic use of communication and social marketing tools to promote positive health outcomes. A nutrition behavior change communication provides individuals with the necessary resources, knowledge, skills, motivation and reinforcement needed to encourage positive change in health behavior [21]. This protocol was developed according to the guidelines of the Consolidated Standards of Reporting Trials (CONSORT) statement extension for cluster randomized trials [22]. A behavior change communication intervention (BCC) is that interacts with the school communities in order to understand their barriers to change and work with them and other family members to seek commitments to change. In adolescent age groups targeted with behavior change strategies that encourage them to adopt dietary diversity and prevention of micronutrient deficiencies on knowledge, attitude and practices before they become mothers. Data obtained from this study are relevant to both governmental and nongovernmental organizations interested in improving the child health and nutrition of an important and often neglected segment of the population in developing and emerging countries. To the best of the investigators knowledge, there is no cluster RCT study conducted in Ethiopia to evaluate the effect of nutrition behavior change communication (BCC) interventions on improving nutritional status and academic performance of schoolchildren. In essence, this cluster randomized controlled trial evaluates a crucible to see how nutritional behavior change communication interventions interact when provided with the nutrition education based on essential nutrition actions 
Citation: Wolde T, Belachew T (2018) A School-Based Cluster Randomized Controlled Trial to Evaluate the Effectiveness of Nutrition Behavior Change Communication Interventions in Improving Nutritional Status and Academic Performance of Schoolchildren in South Ethiopia: Study Protocol. J Nutr Disorders Ther 8: 240. doi:10.4172/2161-0509.1000240

Page 3 of 9

(ENAs) packages and for twelve months thereafter in school settings to affect nutritional status and academic performance of the schoolchild at 10-15 years of age compared to the control arm where routines practices will run unrestricted.

\section{Study aim and hypothesis}

This study protocol aims to test the effectiveness of the nutrition behavioral change communication (BCC) intervention on improving nutritional status (height-for-age $\mathrm{Z}$ score and BMI-for-age $\mathrm{Z}$ score), dietary diversity score (DDS), nutrition knowledge, attitude \& practice (KAP) and academic performance of schoolchildren at 10-15 years age. We hypothesized that our BCC intervention would cause a change of at least 0.3 in mean score of HAZ, BAZ, DDS, KAP and overall average grades of children in intervention arm compared to the control arm.

\section{Methods}

\section{Study design}

This school-based cluster randomized trial (c-RCT) compares the intervention arm with control arm. The intervention arm receives behavior change communication (BCC) on essential nutrition actions. The control arm receives only routine health and nutrition services. From a Meskan District, 10 clusters were selected and randomly assigned to the two study arms by randomization (Figure 1). The schoolchild will be followed-up over the intervention period of 12 months starting from recruitment period.

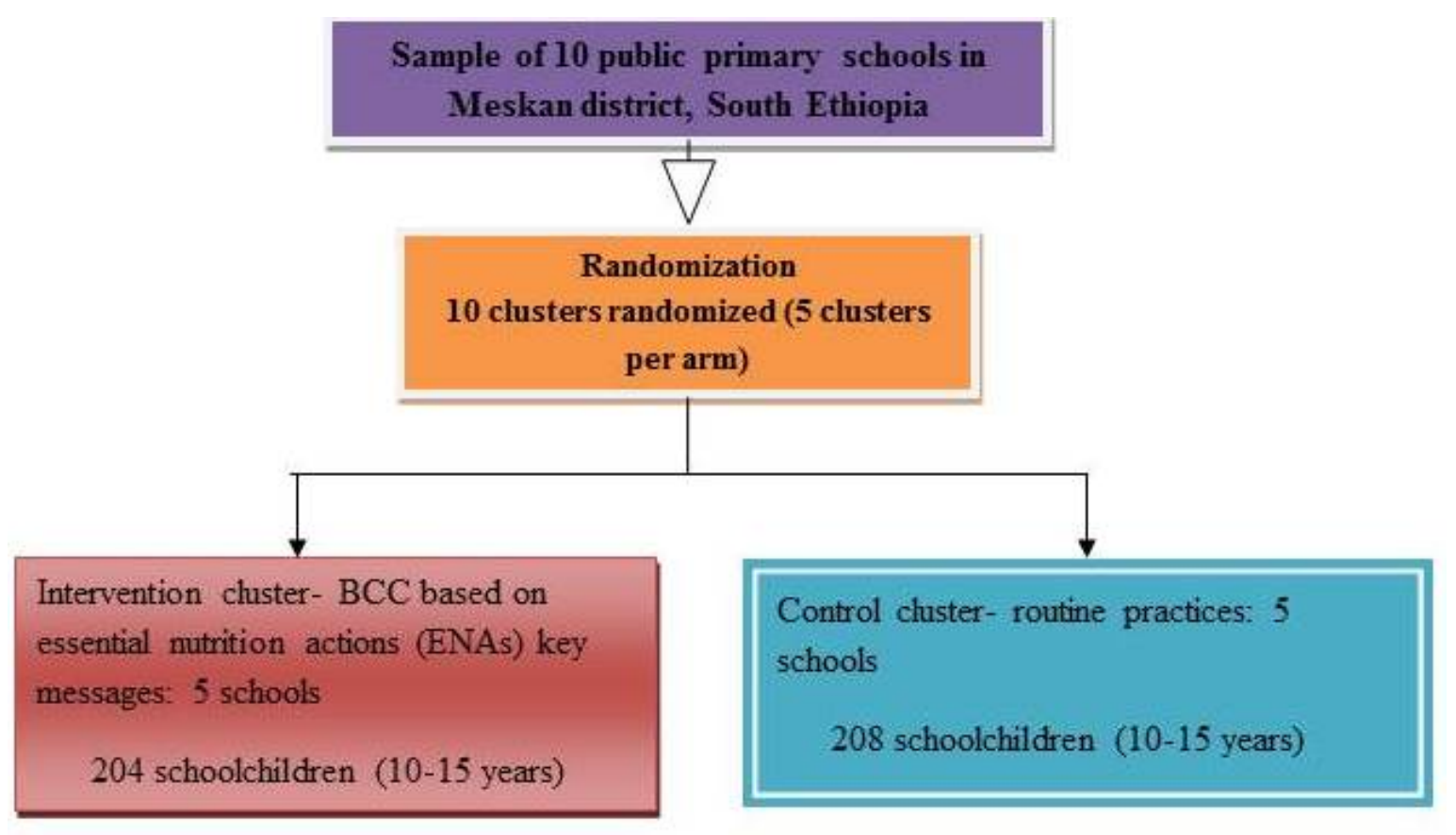

Figure 1: Schematic presentation of randomization.

\section{Study setting}

The study is being conducted in the primary schools of Meskan District, Guraghe Zone, within the Southern Ethiopia where malnutrition prevalence has remained stagnant over a decade. The prevalence of household food insecurity was highest and the prevalence of household food deficit was second highest in Meskan District from the region. Further, up to 150 malnutrition cases are reported monthly from all health facilities of the district [23].

\section{Study population with eligibility criteria}

During the recruitment period, the source population included all primary school children in Meskan district, with schoolchildren from randomly selected primary schools constituting the study population. Primary school children aged 10-15 years, who did not intend to transfer schools during the intervention period, were included. Exclusion criteria included; schoolchildren with obvious congenital or chronic abnormalities impaired feeding or physical growth measurements, edema, and severe illness or clinical complications warranting hospitalization. We excluded schoolchildren whose parents did not provide written consent or were unwilling to participate.

\section{Sample size determination and sampling}

The sample size (n) was determined by using G power statistical software version 3.1 .5 by assuming the effect size of 0.3 , the level of significance $(\alpha), 0.05(\mathrm{Za} / 2=1.96)$, the power of $80 \%(1-\beta=0.84)$ [24], and a dropout rate of $5 \%$. The study included 408 grade 5-8 schoolchildren enrolled in primary schools in Meskan district.

Ten primary schools were selected from the forty primary schools using proportional to size (PS) allocation method. Number and list of students were obtained from school. From each selected schools study participants were selected by simple random sampling based on sampling frame existing in the schools (students' roster). A schematic representation of participant selection is shown in Figure 2. Exclusion criteria for clusters/schools: similar nutrition interventions currently 
Citation: Wolde T, Belachew T (2018) A School-Based Cluster Randomized Controlled Trial to Evaluate the Effectiveness of Nutrition Behavior Change Communication Interventions in Improving Nutritional Status and Academic Performance of Schoolchildren in South Ethiopia: Study Protocol. J Nutr Disorders Ther 8: 240. doi:10.4172/2161-0509.1000240

Page 4 of 9

being implemented by either government or non-government agencies in the selected cluster/school and the cluster/school is too hard to reach.

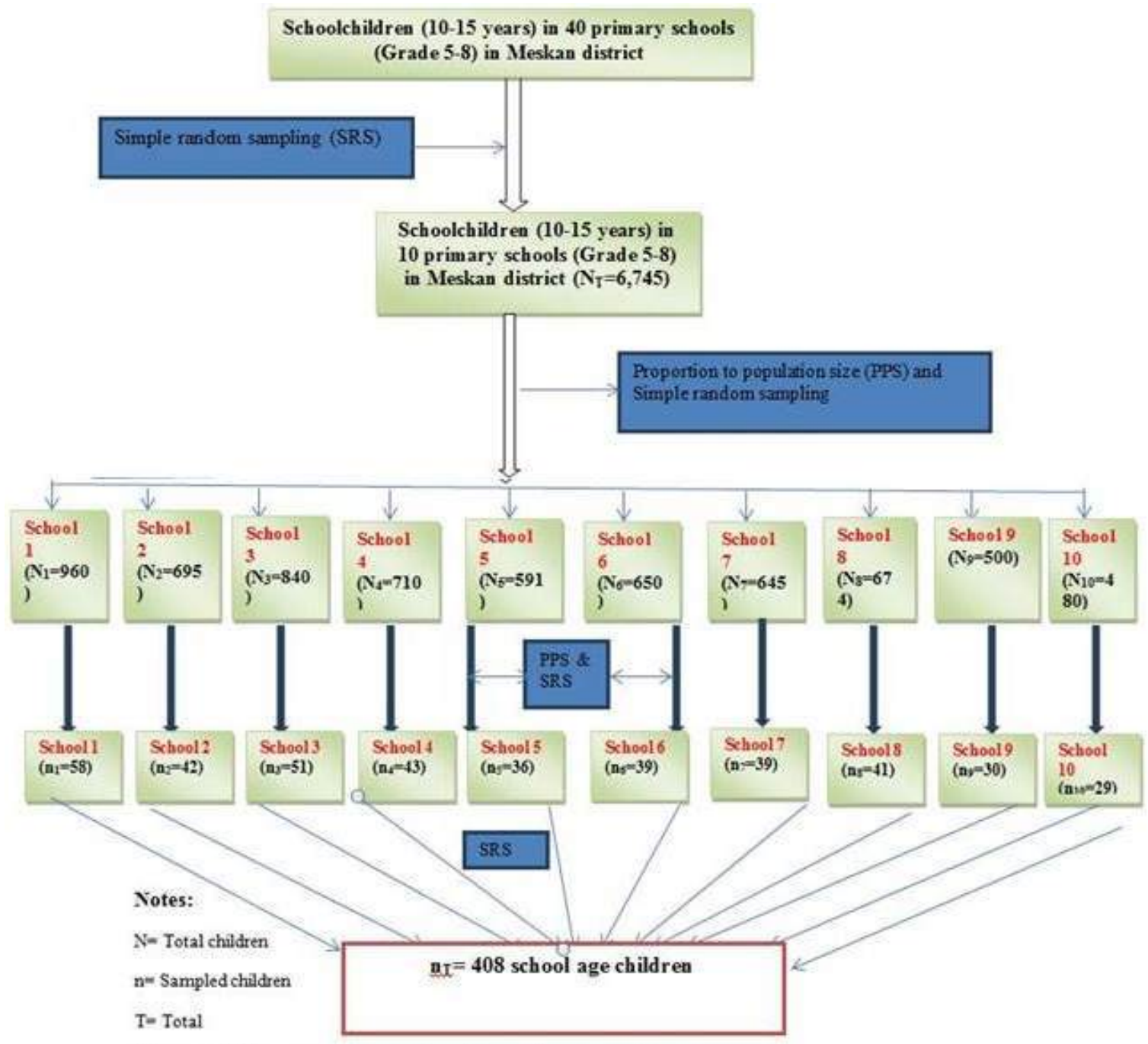

Figure 2: Schematic presentation of participant enrollment.

\section{Randomization}

There are a total of $\mathrm{K}=10$ study schools (clusters), $\mathrm{k}=5$ clusters used in each of the two groups. The intervention and control group allocated randomly to the study schools (clusters) by using a table of random numbers. The randomization process was done as follows; A number 1 to $\mathrm{K}=10$ assigned to each of the study schools (clusters) and then a random starting point located in a table of random numbers by a randomization manager who was not know anything about neither the intervention nor the control schools (clusters) using the random starting point $\mathrm{K}=10$ distinct three digit less likely to include ties than one or two digit numbers. The $\mathrm{K}=10$ distinct three digit random numbers ranked either in ascending or descending order. Then the ranks divided in to two groups (the intervention and control), each costing of $\mathrm{K}=5$ (clusters per each arm) numbers, according to the sequence in which the random numbers appeared. Finally, the two groups assigned to the $\mathrm{K}=10$ study schools (clusters), by using the group numbers as the intervention and control group, and the corresponding rank in each group as the study schools (clusters) to them the corresponding intervention and control group assigned.

\section{Intervention and follow up}

The nutrition behavior change communication (BCC) modules are adapted from the WHO-UNICEF published 'Key messages booklet on Essential Nutrition Actions Framework' with linguistic help from the nationally adapted and validated BCC modules [25].

The provision of the intervention and follow up of the study subjects will take place twelve months between January 1, 2017 and March 31, 2018. The study will have two groups where in the intervention schools; schoolchildren age 10-15 years will receive behavioral change communication (BCC) interventions based poster message on Essential Nutrition Actions (ENAs) standard protocol with the 
Citation: Wolde T, Belachew T (2018) A School-Based Cluster Randomized Controlled Trial to Evaluate the Effectiveness of Nutrition Behavior Change Communication Interventions in Improving Nutritional Status and Academic Performance of Schoolchildren in South Ethiopia: Study Protocol. J Nutr Disorders Ther 8: 240. doi:10.4172/2161-0509.1000240

Page 5 of 9

nutrition education training for twelve months thereafter through school based follow up. While the control schools; schoolchildren age 10-15 years will not receive any interventions for twelve months thereafter through school based follow up.

A guiding nutrition education module and poster with relevant messages on schoolchild promotion of vitamin $\mathrm{A}$, iron \& iodine rich foods to prevent major micronutrient deficiencies will be prepared. The module and poster will be prepared covering the above mentioned topics with detail schedule of delivery for the provision of uniform training across all intervention schools. Poster contains BCC message on vitamin $\mathrm{A}$, iron \& iodine rich foods to prevent major micronutrient deficiencies. Nutrition education will be delivered to students and science teachers every three months by a nutritionist with background of health education. Trained science teachers who will be recruited from the school will facilitate BCC key message at a regular class schedule during the school stay of the schoolchildren and at home after school memorized poster BCC key messages for the period of twelve months. At school level, schoolchildren will remind by science teachers at the end of class session for ten minutes. The school visit will be undertaken weekly for the first month and quarterly for the remaining eleven months follow up. In these school visits, the study teams will facilitate a half-hour individual poster BCC message session for schoolchildren in intervention group. The follow up of all schoolchildren will be undertaken by science teachers and study teams.

\section{Data Collection Tools}

Socio-demographic data; structured questionnaire was used to collect detail data on socio-demographic conditions (age, sex, grade, place of residence, parents education, parents occupation, and family size), household assets, and housing condition. Data collected from schoolchild academic performance record and anthropometric measurement. Schoolchildren' knowledge, attitude \& practice (KAP) towards micronutrient deficiencies prevention and data on food frequency, dietary diversity and sanitation related issues were collected. The questionnaire was also used to collect data on psychosocial factors, preceding illness in the past two weeks, and history of parasitic infections. A structured questionnaire was developed by reviewing relevant literature, such as the nutrition baseline survey report for the National Nutrition Program of Ethiopia by Ethiopian Health and Nutrition Research Institute (EHNRI) [9]. The questionnaire was first prepared in English, then translated into the local language (Amharic), and back translated into English by language experts to check for consistency. Four nurse data collectors and two health officer supervisors were trained for data collection and supervision. Data collectors were trained on measurement of height, basic interview and data collection procedure. The questionnaire was pre-tested and refined on the basis of the feedback obtained from the pre-test.

\section{Outcome Measurements}

The trial intervention was delivering at the school/cluster level will the outcomes of the intervention will be measured at the schools or clusters level. The primary outcome of the study will be improved schoolchildren's dietary diversity score (DDS) and KAP towards micronutrient deficiencies prevention. While the secondary outcomes will includes nutritional status (underweight \& stunting) and academic performance of schoolchildren.

Nutritional Status; Anthropometric measurement will be undertaken for all schoolchildren at baseline and at the end of twelve months follow up. For weight measurement, study subjects remove their shoes, remove their jackets and wear light clothing. Triplicate measurement of weight will be taken at the same day from each study subject using calibrated equipment and standardized techniques. Weight will be measured to the nearest $0.1 \mathrm{Kg}$ with children wearing very light underwear. Electronic SECA scales with a digital screen will be used to measure weight. Height will be measured by making schoolchild lay on flat surface, head position firmly against the fix hardboard, with the eyes looking vertically. The knees extend, by applying firm pressure and feet are flexing at right angles to the lower legs on the board. Height will be measured between the two boards to the nearest accuracy $0.1 \mathrm{~cm}$.

Measurements of height and weight will be taken according to the WHO's guideline [26,27]. Weight of each participant will be measured using standardized and calibrated equipment. To avoid variability/inter examiner error among the data collectors, anthropometric measurements will be taken by the supervisor.

\section{Dietary diversity}

The categories for dietary diversity were determined by first asking if the schoolchildren had eaten a particular type of food in the previous $24 \mathrm{~h}$ prior to date of data collection.

These were then combined into different food groups. Dietary diversity score (DDS) of schoolchildren was assessed and scored as "poor" for 0-3 food group, as "medium" for 4-5 food group and as "high" for greater than 6 food group.

Academic performance; student academic status assessment will be undertaken for all schoolchildren at baseline and at the end of twelve months follow up. The proportion of schoolchildren that will have good academic performance within twelve months follow up period will be recorded and compared in either of the study group.

\section{Quality Control}

Data collectors will check all data collection forms for completeness and consistency before submitting to the supervisor. The school level coordinator will manually checked all of the forms for both completeness and consistency before submitting the forms to the project coordinator who will review again before submitting for data entry to the data clerk. The collected data will be entered by two independent data entry operators. Data quality will be ensured by matching the two sets of entries and verifying records. All anthropometric measurements will be standardized at the beginning of data collection and thereafter periodically by using methods described by WHO.

\section{Data management and statistical analysis}

The baseline data were entered in double, cleaned and checked for missing values and outliers, and analyzed using IBM SPSS statistics for windows, version 23.0. Statistical analysis will be managed by SPSS statistical software Version 23 and also other statistical software's (R) will be considered whenever required. Proportions, percentages, tables, measures of central tendency together with measures of dispersions and graphs will be used to summarize the collected data.

For the purpose of analysis, categorical and numerical scores will be calculated for the academic performance measures. Annual average scores will be calculated by taking the result of two consecutive semesters of 2015-2016. From the anthropometric data, the 
Citation: Wolde T, Belachew T (2018) A School-Based Cluster Randomized Controlled Trial to Evaluate the Effectiveness of Nutrition Behavior Change Communication Interventions in Improving Nutritional Status and Academic Performance of Schoolchildren in South Ethiopia: Study Protocol. J Nutr Disorders Ther 8: 240. doi:10.4172/2161-0509.1000240

Page 6 of 9

undernutrition level (stunting \& underweight) among the schoolchildren at the scheduled measurement of the study will be computed using the standard classification as expressed below; the $\mathrm{z}$ score value for height-for-age and was calculated using the WHO AnthroPlus software [28]. Calculated height-for-age $\mathrm{z}$ score (HAZ) and BMI-for age $\mathrm{z}$ score (BAZ) will be used to classify the level of stunting and underweight using the WHO new reference values for school boys and girls respectively [29]. Children whose HAZ and BAZ are <-2 SDs of the median of the reference population will be considered stunted and underweight respectively. A binary logistic regression analysis will be used to identify independent predictors of nutritional status and academic performance of schoolchildren after controlling for confounding variables.

In order to assess the overall effect of the intervention by controlling other potential contributing factors, a generalized linear mixed model (GLMM) that takes into account the correlated nature of the repeated measurements from each study subject and also from hierarchical structure of data, that study subjects nested in schools (clusters) will be used. This model is flexible to address the different outcome variables to be measured in this study. In all the analysis variables with a p-value $<0.05$ will be considered statistically significant. The presentation will be followed the CONSORT statement guidelines for cluster randomized trials [22].

\section{Data safety steering committee}

We will establish Steering Committee comprising of various professionals including biostatistician, health education, public health expert, nutritionist and the school director to regularly monitor the overall process of the research and intervention activities. The committee will meet every three months to discuss with the research team on the performance of research activities and review the progress.

\section{Ethical consideration}

The study protocol was submitted to Jimma University College of Health Sciences Institutional Review Board for Ethical approval and prior permission will be obtained from the all participating institutions including Regional, Zonal, District Health Offices and District Education Office. The ethical clearance was obtained from the Institutional Ethical Review Committee of College Health Sciences, Jimma University (ref: RpGe/4086/2016). A letter of permission was obtained from the Meskan District education office. School entrance permission was also obtained from each school principal. Informed written consent was obtained from all parents (mothers/caregivers) after explanation of the study objectives, study period and measurement procedures. The verbal assent of the schoolchildren was also asked in addition to parents' consent. The schoolchildren and parents were assured that the information they provided would be kept confidential.

\section{Results}

Out of 408 primary school children in the study; however, 30 schoolchildren (17 boys and 13 girls) declined participation, leaving 378 (93\%) school children paired with their parents from ten primary schools. The mean age of schoolchildren was 12.8 years $(\mathrm{SD} \pm 1.3)$ and the average size of the family was $6.8(\mathrm{SD} \pm 1.9)$. The girl-boy ratio was 1.21 with 207 (54.8\%) girls were involved into the study. Majority of the study participants $273(72.2 \%)$ were from families who were currently on residing in rural areas. Two hundred five (54.2\%) of them had fathers who attended primary school. Two hundred twenty eight (60.3\%) had had fathers whose occupation was farming. One hundred five $(27.8 \%)$ of respondents were categorized in the fourth wealth quintile (Table 1).

\begin{tabular}{|c|c|c|c|}
\hline Variables & Categories & Frequency & Percent \\
\hline \multirow[b]{2}{*}{ Sex } & Boy & 171 & 45.2 \\
\hline & Girl & 207 & 54.8 \\
\hline \multirow[b]{2}{*}{ Age in years } & 10-Dec & 159 & 42.1 \\
\hline & $13-15$ & 219 & 57.9 \\
\hline \multirow[b]{2}{*}{ Place of residence } & Rural & 273 & 72.2 \\
\hline & Urban & 105 & 27.8 \\
\hline \multirow[b]{4}{*}{ Father's educational status } & Illiterate & 125 & 33.1 \\
\hline & Primary complete & 205 & 54.2 \\
\hline & Secondary complete & 41 & 10.8 \\
\hline & Above secondary & 7 & 1.9 \\
\hline \multirow[b]{4}{*}{ Mother's educational status } & Illiterate & 189 & 50 \\
\hline & Primary complete & 167 & 44.2 \\
\hline & Secondary complete & 18 & 4.8 \\
\hline & Above secondary & 4 & 1.1 \\
\hline \multirow[b]{4}{*}{ Father's occupation } & Farmer & 228 & 60.3 \\
\hline & Government employee & 28 & 7.4 \\
\hline & Merchant & 71 & 18.8 \\
\hline & Other & 51 & 13.5 \\
\hline \multirow[b]{5}{*}{ Mother's occupation } & Household work & 235 & 62.2 \\
\hline & Government employee & 9 & 2.4 \\
\hline & Farmer & 16 & 4.2 \\
\hline & Merchant & 103 & 27.2 \\
\hline & Other & 15 & 4 \\
\hline \multirow[b]{5}{*}{ Wealth quintile } & Lowest & 76 & 20.1 \\
\hline & Second & 72 & 19 \\
\hline & Middle & 61 & 16.1 \\
\hline & Fourth & 105 & 27.8 \\
\hline & Highest & 64 & 16.9 \\
\hline \multirow[b]{2}{*}{ Family size } & $<7$ & 172 & 45.5 \\
\hline & $>=7$ & 206 & 54.5 \\
\hline
\end{tabular}

Table 1: Socio-demographic and economic characteristics of respondents at primary schools of Meskan district, South Ethiopia, October 2016. 
Citation: Wolde T, Belachew T (2018) A School-Based Cluster Randomized Controlled Trial to Evaluate the Effectiveness of Nutrition Behavior Change Communication Interventions in Improving Nutritional Status and Academic Performance of Schoolchildren in South Ethiopia: Study Protocol. J Nutr Disorders Ther 8: 240. doi:10.4172/2161-0509.1000240

Page 7 of 9

Anthropometric and academic assessment of students

\begin{tabular}{|c|c|c|c|c|}
\hline Variables & Boy++ & Girl++ & $\mathbf{p}^{*}$ & Mean difference \\
\hline Age in years & $12.61 \pm 1.29$ & $12.94 \pm 1.23$ & 0.012 & -0.33 \\
\hline Height in $\mathrm{cm}$ & $153.86 \pm 9.14$ & $155.36 \pm 8.89$ & 0.107 & -1.5 \\
\hline Height-for-age (HAZ) & $-0.014 \pm 1.14$ & $0.047 \pm 1.17$ & 0.613 & -0.06 \\
\hline Overall average grades score & $64.01 \pm 8.38$ & $64.93 \pm 8.49$ & 0.292 & -0.92 \\
\hline English score & $59.24 \pm 11.86$ & $60.11 \pm 12.21$ & 0.488 & -0.87 \\
\hline Math's score & $57.88 \pm 9.61$ & $58.86 \pm 11.10$ & 0.366 & -0.98 \\
\hline Class rank & $26.52 \pm 17.01$ & $25.29 \pm 15.66$ & 0.463 & 1.24 \\
\hline
\end{tabular}

Table 2: Anthropometric and academic performance of schoolchildren by gender at primary school of Meskan district, Guraghe Zone, South Ethiopia, October 2016.

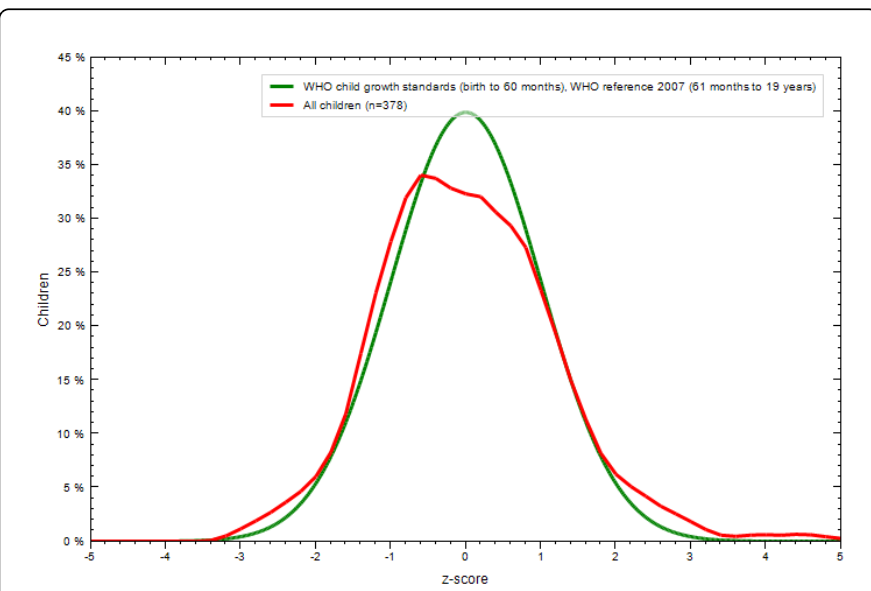

Figure 3: Height for age $\mathrm{z}$ score of students at primary schools of Meskan district, Guraghe Zone, South Ethiopia, October 2016.

Anthropometric measurements; height and weight were taken and the nutritional index, height-for-age and BAZ were derived to assess the nutritional status of students. Mean height and weight of the study participants were $154.68 \mathrm{~cm}(\mathrm{SD} \pm 9.02 \mathrm{~cm})$ and $41.56 \mathrm{Kg}(\mathrm{SD} \pm 8.31$ $\mathrm{Kg}$ ) respectively. Nutritional index that are derived from anthropometric data indicated that prevalence of stunting and underweight in the study participants were $64(16.9 \%)$ and $141(37.3 \%)$ respectively (Table 2, Figures $3 \& 4$ ).

The association between different variables was tested for their association with stunting in binary logistic analysis (Table 3). Children whose were living in rural residence $(\mathrm{COR}=2.2 ; 95 \% \mathrm{CI}=1.26,3.86)$, having own land for agricultural $(\mathrm{COR}=2.2 ; 95 \% \mathrm{CI}=1.01,4.94)$, availability of home animal $(\mathrm{COR}=2.4 ; 95 \% \mathrm{CI}=1.04,5.56)$, and home comfortable to study $(\mathrm{COR}=0.2 ; 95 \% \mathrm{CI}=0.05,0.84)$ were associated with stunting.

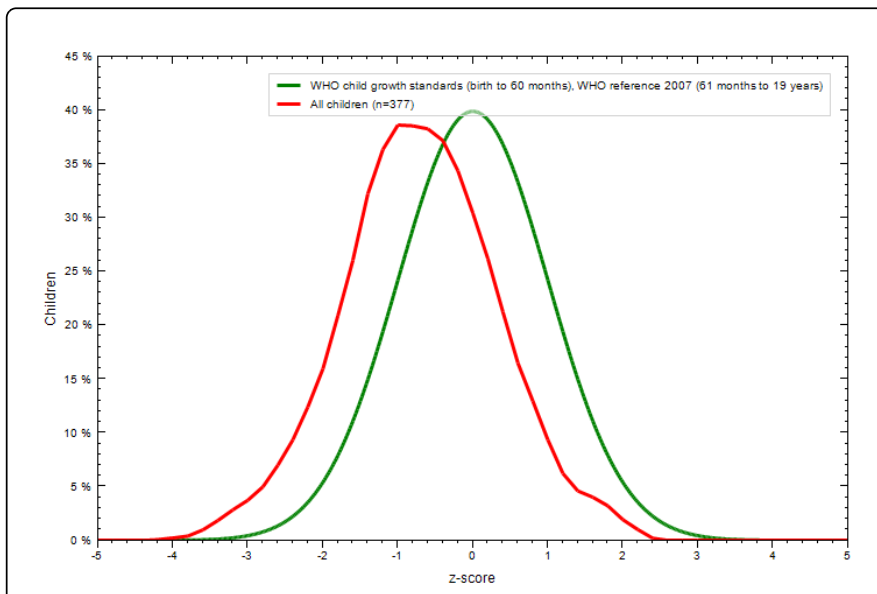

Figure 4: BMI for age $\mathrm{z}$ score of students at primary schools of Meskan district, Guraghe Zone, South Ethiopia, October 2016.

Sex, age, educational status, occupation, family size, relative household wealth index, average grades score, math's score, English score, psychosocial conditions of schoolchildren and parental involvement in schooling variables included in the study were not associated significantly with stunting $(\mathrm{p}>0.05)$.

All variables that have association (at significance level of 0.05) with outcome variable in the bivariate analysis were included in the multivariate regression model. Place of residence (AOR=1.97; $95 \% \mathrm{CI}=1.12,3.46)$ had independent association with stunting. School children from the area of rural residence were 2 times more likely to be stunted than those who are living in urban residence (Table 3 ). 
Citation: Wolde T, Belachew T (2018) A School-Based Cluster Randomized Controlled Trial to Evaluate the Effectiveness of Nutrition Behavior Change Communication Interventions in Improving Nutritional Status and Academic Performance of Schoolchildren in South Ethiopia: Study Protocol. J Nutr Disorders Ther 8: 240. doi:10.4172/2161-0509.1000240

Page 8 of 9

\begin{tabular}{|c|c|c|c|c|}
\hline \multirow[b]{2}{*}{ Variables } & \multicolumn{2}{|c|}{ Stunted } & \multirow{2}{*}{$\begin{array}{l}\text { Crude OR } \\
95 \% \mathrm{Cl}\end{array}$} & \multirow{2}{*}{$\begin{array}{l}\text { Adjusted OR } \\
95 \% \mathrm{Cl}\end{array}$} \\
\hline & Yes & No & & \\
\hline \multicolumn{5}{|c|}{ Residence } \\
\hline Urban & 37 & 236 & 1 & 1 \\
\hline Rural & 27 & 78 & $2.208(1.263-3.858)$ & $1.967(1.119-3.457)^{*}$ \\
\hline \multicolumn{5}{|c|}{ Own land for agricultural } \\
\hline Yes & 54 & 290 & $2.238(1.013-4.944)$ & $1.613(0.659-3.947)$ \\
\hline No & 10 & 24 & 1 & 1 \\
\hline \multicolumn{5}{|c|}{ Availability of home animal } \\
\hline Yes & 55 & 294 & $2.405(1.041-5.559)$ & $1.405(0.532-3.713)$ \\
\hline No & 9 & 20 & 1 & 1 \\
\hline \multicolumn{5}{|c|}{ Home comfortable to study } \\
\hline Yes & 62 & 270 & $0.198(0.047-0.839)$ & $0.269(0.062-1.165)$ \\
\hline No & 2 & 44 & 1 & 1 \\
\hline
\end{tabular}

Table 3: Factors associated with stunting at primary schools of Meskan district, Guraghe Zone, South Ethiopia, October 2016.

\section{Discussion}

Previous evidence suggests that behavior change communication (BCC) interventions can improve health related outcomes, including Mother and child Health (MCH) indicators. For example community based and culturally accepted educational interventions, were shown to improve complementary feeding practices (Alive \& Thrive partner with $\mathrm{FMOH}$ ), dietary intake, and growth [30]. Interventions such as counseling, targeted towards improving maternal nutrition, uptake of iron and folic acid and other nutrients; breast feeding practices, can improve outcomes for $\mathrm{MCH}$ [31]. A report by USAID described that effectiveness of BCC strategy is very strong especially in improving breast feeding [32]. However, behavior change communication interventions of school children about improved micronutrient intake on nutritional status and academic performance received little programmatic and research attention in developing countries including Ethiopia.

Behavior Change Communication (BCC) intervention can be used to improve health reduces disease risks, manage chronic illness and improve the overall well-being of individuals, families and communities. However as we know, not every intervention is successful. It has been demonstrated that the ones most likely to succeed are those that are based on a very clear understanding of the targeted health behavior and the environmental context. There are many theories of health behavior and these theories have been helpful when planning, implementing and evaluating behavior change communication intervention.

In an effort to accelerate the reduction of micronutrient malnutrition, BCC intervention has not been implemented in school age children in many countries in sub Saharan Africa (SSA) including in Ethiopia. It is well-accepted that decisions regarding BCC in nutrition intervention should be based on robust evidence of the benefits and cost-effectiveness of the intervention. The intervention protocol will be implemented for a period of twelve months, after which anthropometric, nutritional knowledge, diet diversity scores and academic performance will be determined.

The strength of our study is that, to our knowledge, this is the first study protocol with a cluster school based RCT that is examining the impact, advantages and optimal duration of BCC intervention for students, and science teachers. A limitation of our study includes only assess anthropomorphic measurements and did not assess the micronutrient status of study participants. Furthermore, there may have been differences in the evaluation system for schoolchildren academic performance among the study schools. Physical performance capacity and motor skills were not measured in this study.

The findings of this study will provide sufficient evidence to develop policies and programs aimed to improve dietary diversity in children as well as nutritional knowledge (10-15 years) and to prevent micronutrient deficiencies and poor academic performance in school settings. It will also provide recommendations for strengthening the school nutrition component of behavior change communication in child health and nutrition programs.

\section{Trial status}

Recruitment and baseline data has been completed. Intervention and end line data collection is ongoing.

\section{Acknowledgements}

The authors would like to thank the Zonal Health district of Health and Education Bureaus and their contributions to successfully launch the implementation of this study protocol. The authors acknowledge the Ethiopian Public Health Institute (EPHI) for the technical and 
Citation: Wolde T, Belachew T (2018) A School-Based Cluster Randomized Controlled Trial to Evaluate the Effectiveness of Nutrition Behavior Change Communication Interventions in Improving Nutritional Status and Academic Performance of Schoolchildren in South Ethiopia: Study Protocol. J Nutr Disorders Ther 8: 240. doi:10.4172/2161-0509.1000240

Page 9 of 9

logistical support to the study. We would also like to thank the school directors, teachers and study data collectors for their efforts and especially the study participants for their time and support of this study.

\section{Authors' Contributions}

TW and TB conceptualized the study protocol. TW and TB contributed in selection of the interventions and initial study design. TW and TB contributed during the finalization of the study design. TW led writing of the study protocol with significant inputs from all co-authors. TW and TB developed the intervention and assessment tools. All authors contributed to the development, review and finalization of the study protocol manuscript.

\section{Competing Interests}

All authors declare that they have no competing interests.

\section{References}

1. The World Bank. Repositioning Nutrition as Central to Development A Strategy for Large-Scale Action, Washington, DC 20433 USA, 2006, 272p.

2. [No authors listed] (2015) Federal Democratic Republic of Ethiopia $\mathrm{MOH}$, National Nutrition Program.

3. Michael C, Latham MB, DTM, FAPHA, Cobos F (1971) The Effects of Malnutrition on Intellectual Development and Learning 61: 1307-1324.

4. Chinyoka K (2016) Impact of Poor Nutrition on the Academic Performance of Grade Seven learners囚: A Case of Zimbabwe.

5. [No authors listed] (2009) Federal Democratic Republic of Ethiopia $\mathrm{MOH}$. The Cost of Hunger in Ethiopia "The Social and Economic Impact of Child Under nutrition in Ethiopia Summary Report" Implications for the Growth and Transformation of Ethiopia.

6. Emerick L (1992) Academic underachievement among the gifted Students' perceptions of factors that reverse the pattern 36: 140-146.

7. [No authors listed] (2005) Symposium on developmental and behavioral disorders-II: Poor school performance 72 (11).

8. Levinger B (1992) Nutrition health and education, current issue and trends. International basic education programs.

9. [No authors listed] (2009) National Nutrition Baseline Survey for the National Nutrition Program of Ethiopia. Ethiopian Health and Nutrition Research Institute, Addis Ababa.

10. Bidu KT, Hailemariam T, Negeri EL (2016) Prevalence and associated factors of undernutrition among school adolescents in Gobu Seyo District, East Wollega Zone, Oromia regional state of West Ethiopia 10: 251-269.

11. Yebyo HG, Birhane K, Gesesew HA (2015) Assessment of Adolescents Under Nutrition Level among School Students in Assessment of Adolescents' Under Nutrition Level among School Students in Eastern Tigray, Ethiopia囚: A Cross-Sectional Study.

12. Mekonnen H, Tadesse T, Kisi T (2013) Malnutrition and its Correlates among Rural Primary School Children of Fogera District, Northwest Ethiopia Nutritional Disorders \& Therapy S12: 002.

13. Woday A, Menber Y, Tsegaye D (2018) Prevalence of and Associated Factors of Stunting among Adolescents in Tehuledere District, North East Ethiopia, 2017. J Clin Cell Immunol 9: 546.
14. Birru SM, Belew AK, Tariku A (2018) One in three adolescent schoolgirls in urban northwest Ethiopia is stunted 1-8.

15. Melaku YA, Zello GA, Gill TK, Adams RJ, Shi Z (2015) Prevalence and factors associated with stunting and thinness among adolescent students in Northern Ethiopia®: a comparison to World Health Organization standards. Arch Public Health 1-11.

16. Assefa H, Belachew T, Negash L (2015) Socio-demographic factors associated with underweight and stunting among adolescents in Ethiopia. Pan African Medical Journal 20: 252

17. Berheto TM, Mikitie WK, Argaw A (2015) Urban-rural disparities in the nutritional status of school adolescent girls in the Mizan district, southwestern Ethiopia 1-10.

18. Ministry of Education (2012) National School Health and Nutrition Strategy. Addis Ababa: Ministry of Education.

19. Haile D, Nigatu D, Gashaw K, Demelash H (2016) Height for age z score and cognitive function are associated with Academic performance among schoolchildren aged 8-11 years old Archives of Public Health. 74:17

20. Ethiopian Ministry of Education. Education statistics Annual Abstract.

21. Sydner L (2007) Health Communication Campaigns and Impact on Behavior. Journal of Nutrition Education and Behavior 39: 32-40.

22. Campbell M, Piaggio G, Elbourne D, Altman D (2012) CONSORT 2010 statement: extension to cluster randomized trials. Br Med J 345: e5661e5671.

23. [No authors listed] (2006) Emergency Nutrition Coordination Unit Disaster Prevention and Preparedness Agency. Emergency nutrition quarterly bulletin.

24. Faul F, Erdfelder E, Lang A, Buchner A, Power G (2007) A flexible statistical power analysis program for the social, behavioral, and biomedical sciences. Behavior research method.

25. Essential-nutrition-actions-and-essential-hygiene-actions-framework [Available at http://www.coregroup.org/resources/488-essentialnutrition-actions-and-essential-hygiene-actions-framework]

26. World Health Organization (WHO) (2009) WHO AnthroPlus for Personal Computers Manual: Software for Assessing Growth of the World's Children and Adolescents. Geneva: WHO.

27. Bruce C (2003) Anthropometric Indicators Measurement Guide. Food and Nutrition Technical Assistance Project, Academy for Educational Development, Washington.

28. World Health Organization (WHO) (2009) WHO AnthroPlus for Personal Computers Manual: Software for Assessing Growth of the World's Children and Adolescents. Geneva: WHO.

29. WHO (2006) Multicentre Growth Reference Study Group. WHO Child Growth Standards: length/height-for-age, weight-for-age, weight-forlength, weight-for-height and body mass index-for-age: methods and development

30. USAID (2011) Alive and Thrive: Scaling Up Infant and Young Child Feeding Programs in Bangladesh, Ethiopia, and Vietnam: A population level behavior change communication strategy.

31. Bhutta ZA, Das JK, Rizvi A, Gaffey MF, Walker N, et al. (2013) Evidence based interventions for improvement of maternal and child nutrition: what can be done and at what cost? Lancet 382: 452-477.

32. Lamstein (2014) Evidence of Effective Approaches to Social and Behavior Change Communication for Preventing and Reducing Stunting and Anemia. 